\title{
Participating in Enthymematic Dialogue
}

Thus far we have focused on how a theory of enthymemes and topoi contributes to semantic and pragmatic interpretation of dialogue. In this chapter we will focus more on how agents engaged in conversation produce dialogue contributions drawing on topoi which they themselves have access to, as well as other dialogue participants' capacity to identify enthymemes and underpinning topoi. First, we will consider the link between enthymemes and cognitive load. We will discuss the notion of redundancy in dialogue in the context of Walker's (1996) research on so called information redundant utterances (IRU s), and how we perceive these utterances as serving to add new information to a discourse situation by pointing to specific topoi.

We will discuss how enthymematic arguments are embedded in conversational games associated with speech act types such as assertion, suggestion, request, etc. We will also consider how we can model the invention of enthymematic arguments, as well as how an enthymematic relation may be accommodated based on accessible topoi.

\section{1 $\quad$ Enthymemes and Cognitive Load}

\subsubsection{Information Redundancy in Dialogue}

A significant feature of natural language-particularly dialogue-is economy. This has been noted by many scholars in the fields of pragmatics and discourse studies, and given rise to some of the well known and generally accepted theories previously discussed in this book. Walker (1996) mentions the second part of Grice's (1975) maxim of quantity, as an example of a generally assumed redundancy constraint, that is, the notion that communicative contributions should not be redundant with regard to informational content.

This maxim, "do not make your contribution more informative than required", has often been interpreted as "make your contribution as short as possible", resulting in all utterances whose content may be deduced from context or retrieved from memory being considered IRU s. Walker (1996) argues that IRU S are often not redundant at all (thus actually adhering to the maxim of quantity rather than violating it). Rather, IRU s serve to help lower the cognitive load of an interlocutor interpreting a dialogue contribution. For example, in (5.1), the 
second part of the utterance is informationally redundant in the sense that the other dialogue participant is likely to be aware of who is president.

(5.1) A: Clinton has to take a stand on abortion rights for poor women. A: HE'S THE PRESIDENT (Walker, 1996, p. 188)

The second part of the utterance above provides the non-redundant information that the fact that Clinton is president is the reason the speaker thinks that he has to take a stand on abortion rights for poor women. As we will demonstrate, many of the utterances that Walker refers to convey enthymemes and are underpinned by topoi.

According to Walker the principle of avoiding redundancy, which has often taken precedence in work on dialogue modelling and overshadowed other factors affecting communicative choice, is based on four assumptions about dialogue:

(5.2) a. Unlimited working-memory: everything an agent knows is always available for reasoning;

b. Logical omniscience: agents are capable of applying all inference rules, so any entailment will be added to the discourse model;

c. Fewest utterances: utterance production is the only process that should be minimised;

d. No autonomy: assertions and proposals by agent $A$ are accepted by default by agent $B$.

Walker presents corpus data in which agents frequently violate the redundancy constraint, indicating that the fewest utterances assumption is not correctsometimes other aspects of communication are more important than economy.

Walker's analysis leads her to formulate three main functions of IRU s:

(5.3) a. To provide evidence supporting beliefs about mutual understanding and acceptance.

b. To manipulate the locus of attention of the discourse participants by making a proposition salient.

c. To augment the evidence supporting beliefs that certain inferences are licensed.

Walker also discusses experiments, which were designed according to the results of the corpus study. These experiments show that IRU s do indeed contribute to mitigating the effect of an agent's resource limits. 
Let us now take a look at one of Walker's examples of an IRU. ${ }^{1}$ The context of this excerpt is two colleagues walking to work. They are both familiar with the surroundings and the routes available.
a. A: Let's walk along Walnut Street
b. A: It's shorter (Walker, 1996, p. 188)

It is known to $A$ that $B$ knows that Walnut Street is shorter, so by the redundancy constraint $A$ should only have said (5.4a). Walker claims that (5.4b) is considered an IRU based on the assumption of unlimited working memory, i.e. that all knowledge and information an agent has access to is equally available at all times. Walker hypothesises that the mentioning of the well-known fact that Walnut Street is shorter is a way for $A$ to ease $B$ 's cognitive load. Another example is (5.5), which is an excerpt of a discussion about individual retirement accounts.

(5.5) a. $A: \mathrm{Oh}$ no, individual retirement accounts are available as long as you are not a participant in an existing pension.

b. $B$ : Oh I see. Well [...] I do work for a company that has a pension.

c. A: Ahh. Then you're not eligible for [the tax year of] eighty one. (Walker, 1996, p. 187)

Walker's analysis of this example is that $(5.5 \mathrm{c})$ is considered an IRU based on the assumption that agents are logically omniscient, since $B$ would have to apply an inference rule to conclude $(5.5 \mathrm{c})$. The function of $A$ 's stating $(5.5 \mathrm{c})$ is, according to Walker, to augment the evidence supporting beliefs that certain inferences are licensed.

\subsubsection{A Rhetorical Approach to IRUs}

A difficult question in this context is how to decide when to add an IRU and when not to. Some redundancy may help relieve the working memory of a dialogue system user or a participant in human-human conversation, while too much information will only increase the cognitive load. We will discuss how a rhetorical perspective may be of use in this balancing act, and suggest that enthymemes, underpinned by topoi, may provide a model for analysing these utterances.

1 This example has been discussed previously in the context of conventional and conversational implicature, Sections 2.2 and 2.3. 
We would like to suggest a way of looking at IRU s which elucidates Walker's ideas about their function, and offers an alternative to the four assumptions of the redundancy constraint. The three functions of IRU s in Walker's study have in common that they aim to lead the listener to a certain conclusion, either by supporting a belief the listener already has, or by directing, or even redirecting, the attention of the listener. In other words, IRU s are rhetorical. Examples (5.4) and (5.5) are both illustrations of this. The fact that (5.4b) is considered redundant according to the redundancy constraint seems to reflect not only the unlimited working memory assumption, but also the assumption that dialogue participants do not have a will of their own in the sense that they by default accept assertions and proposals by other agents. In fact, it is the relative autonomy of $B$ that makes it possible for him not to accept $A$ 's proposition. By providing a reason for choosing Walnut Street, $A$ performs a rhetorical act that potentially increases the likelihood that the suggestion will be accepted by $B$.

Example (5.5) also indicates that $A$ wants to make sure that $B$ draws a specific conclusion. It seems likely that $A$, if she did not find it of some importance that $B$ draws the conclusion $(5.5 \mathrm{c})$, might not bother to make the inference explicit $-B$ could still be expected to make the inference. However, for $B$ to do that would not necessarily make him logically omniscient- the assumption Walker (1996) claims to be the reason for considering (5.5b) an IRU— just capable of making some inferences.

Interestingly, many of Walker's examples of IRUs and their respective antecedents constitute structures similar to that of an enthymeme. The mentioning of one carefully chosen premise directs the attention of the listener in the direction that the speaker wants, and makes the listener a bit more likely to accept the proposition presented in the conclusion. The enthymeme might of course serve to persuade or even mislead a listener, but the same mechanism can also make it easier for a conversational participant to accept an honest and constructive proposal made by another agent. This would be helpful when quick decisions need to be made, or when demanding parallel activities require attention.

Let us go back to the colleagues walking to work. Example (5.4) above could easily be analysed within a rhetorical framework. Mentioning (5.4b) could be a way for $A$ to point to the argument about the shortest route, perhaps because they are running late. There could be other reasons to walk along Walnut Street, perhaps that it is more quiet. $A$ might know that $B$ usually prefers a busy street, but that she does not particularly like to walk, which would make the shortargument more persuasive. If they were not in a hurry, and $A$ wanted them to walk along Walnut Street because it is nicer to walk along a quiet street than 
a busy one, $A$ would probably say 'Let's walk along Walnut Street. It's quieter' thus validating her suggestion.

However, it is also possible that $A$ would want to walk along Walnut Street for some reason that she does not want $B$ to know about. So, by providing the premise "it's shorter", A makes it easier for B to interpret the suggestion to walk along Walnut Street in the intended way, that is, associated with particular inferences and not with others. The provided premise may or may not point to a genuine reason of A's for suggesting Walnut Street. However, it is also possible that A, by supplying the premise, is merely externalising her own way of thinking, not considering B's mental states. For a discussion on externalised inference, see Pickering and Garrod (2004), Mills and Gregoromichelaki (2010) and Gregoromichelaki et al. (2011).

So, giving the premise "it's shorter" points to an argument drawing on certain topoi, without which the utterance would be difficult to make sense of. The "hidden premise", i.e. the premise that $B$ adds to the argument, would be something that makes sense in the context, having to do with for example time (as above) or effort (we don't want to walk longer than necessary). The additional premise is necessary in order to make the enthymeme fit with the relevant topos.

A rhetorical perspective that uses enthymematic arguments as an explanatory model for how information is given and withheld, would be based on a different set of assumptions about dialogue than those Walker formulates as the basis of the redundancy constraint. Thus we propose four rhetorically motivated principles as an alternative to the four principles of economy in dialogue in (5.2):

(5.6) a. Limited working-memory: suggestions help agents to reach a certain decision

b. Logical capacity: agents are capable of applying some inference rules, some entailments will be added to the discourse model;

c. Utterance production: should be balanced so as to maximise persuasion

d. Autonomy: assertions and proposals by agent $A$ are not accepted by default by agent $B$, and different agents may or may not share goals and intentions.

As humans we need reasons to validate propositions we are presented with. We know this intuitively-it is difficult to complete a task if we are just presented with single pieces of information that do not seem to be connected. The same conclusion can be drawn based on different premises, and we often 
want to know which argument the speaker is referring to before we accept a proposition. There are situations where the standard way to instruct is by single utterances (or orders), such as in the military, or in other contexts where the roles are very well defined, and the modus operandi of the activity well rehearsed, such as in surgery.

We agree with Walker's conclusion that IRU s serve to ease cognitive load in different ways. We also suggest that the reason for this is that the enthymematic structure helps the recipient of the IRU to make up her mind, or correctly interpret an utterance. If the IRU provided links the assertion or suggestion made to a topos which the recipient finds acceptable she is more likely to agree with the proposition.

\subsection{Enthymemes and Dialogue Context}

In the previous chapter we discussed what we could call assertion enthymemes, like the one in $(5 \cdot 7)$.

(5.7) Anon 3: the monarchy are non political $\langle$ pause $\rangle$ and therefore, when they choose to speak it's usually out of a genuine concern for that problem.

(BNC: FLE 233)

In (5.7) the speaker claims that when (representatives of) the monarchy speak, it is out of genuine concern, supporting this claim with another claim, that they are non-political. Whether or not this is perceived as an accessible, relevant and acceptable argument by other conversational participants depends partly on to what extent they find that it is consistent with a relevant topos. This enthymeme seeks to establish a certain take on what the world is like.

However, reasoning in dialogue is often reasoning towards an action or decision. Enthymemes in arguments like (5.4) for example, consist of a conclusion or consequent part which conveys a speech act like request or exhortation. They belong to what Aristotle called deliberative rhetoric, which is commonly found in political discourse (Corbett and Connors, 1999).

The goal of deliberative discourse is to convince someone to carry out (or not carry out) a future action. In the context of politics this could be things like go to war, extend the public transport system of a city, or cut taxes. However, everyday conversations also often involve deliberative discourse. Conversational participants have to decide things like who to invite to a party, which car to buy, which restaurant to go to, or which film to see. In the context of deciding, interlocutors present arguments to each other. Also, many of the tasks for which we 
want to use dialogue systems are linked to advice giving, instructing etc. where the point of the system is to advise users based on assembled information and considerations of the context.

In the rest of this chapter we will focus on action-directed enthymemes embedded in a machinery of conversational games, taking as our point of departure the excerpt presented previously in (5.4). We will suggest how conversational games can be used to move a dialogue forward and how the kind of conversational game you choose to carry out a communicative project is related to the rhetorical force of the enthymeme conveyed.

Let us now consider (5.8).

$A$ : Let's walk along Walnut Street.

A: It's shorter.

In (5.8) speaker $A$ uses an enthymematic argument to communicate to another dialogue participant $B$ that they should choose Walnut Street rather than other possible routes, and that the reason for suggesting Walnut Street is that it is shorter than other options. We see this enthymeme in $\varepsilon_{\text {shorter }}$ in (5.9) - a function from a situation of a type where Walnut Street is shorter than some alternative, to a type of situation where $A$ and $B$ walk along Walnut Street. Note that we cannot know the exact nature of the enthymeme in terms of the number of features present, for example the number of routes available to $A$ and $B$.

$$
\varepsilon_{\text {shorter }}=\lambda r:\left[\begin{array}{l}
\mathrm{x}=\text { W.St. }: \text { Ind } \\
\mathrm{y}=\text { other_route }: \text { Ind } \\
\mathrm{z}=\operatorname{SELF}: \text { Ind } \\
\mathrm{w}: \operatorname{set}(\text { Ind }) \\
\mathrm{c}_{1}: \operatorname{route}(\mathrm{x}) \\
\mathrm{c}_{2}: \operatorname{route}(\mathrm{y}) \\
\mathrm{c}_{3}: \operatorname{shorter} \text { than }(\mathrm{x}, \mathrm{y}) \\
\mathrm{c}_{4}: \operatorname{in}(\mathrm{z}, \mathrm{w})
\end{array}\right] \cdot[\mathrm{e}: \text { walk_along }(r . \mathrm{w}, r . \mathrm{x})]
$$

Using an enthymeme like the one in (5.9) is essentially a conversational strategy for carrying out a communicative project. In Chapter 3 we discussed the gameboard feature "project" and how we relate it to "communicative activity".

When an activity is carried out, it is made up of a number of projects, the goals of which are ideally fulfilled during the course of the interaction. For example, the activity type "medical consultation" typically includes a number of projects which need to be carried out, such as establishing the patient's symptoms, diagnosing the patient and explaining to the patient how his condi- 
tion should be treated (Berbyuk Lindström, 2008). The activity "chatting with neighbour while gardening" is less formal, and it is not strongly associated with particular projects to be carried out in a specific order. However, if we were to examine data from this activity, we would be likely to identify communicative projects regarding things like current affairs, the weather, gardening, etc. This does not mean that the agents involved in the conversation have anticipated any of these projects, as would probably be the case in the medical consultation scenario.

Activity types also differ in terms of to what extent the roles are set or institutionalised. In the case of medical consultation, certain behaviour in the carrying out of a project is closely related to an activity role like "patient". In many activity types, however, activity roles do not play an important part for the order in which dialogue participants are expected to make their contributions. Neither do they always affect the type of contribution which we expect from a particular dialogue participant. However, even in an informal activity there are role related requirements for participating in the activity. For example, for participating in an informal conversation between friends, you have to know the other conversational participants, and the role "friend" comes with certain obligations like being honest, supportive and kind. However, assuming the role of "friend" is not associated with a particular pattern of conversation, as is the case when assuming the role of "doctor" or "patient" in a medical consultation conversation.

\subsection{Conversational Games}

As we mentioned briefly in Section 3.2.3, we consider the carrying out of a communicative project to be associated with a set of conversational games. The notion of dialogue game is well established in research on dialogue and is described for example in Carlson (1982) and Levin and Moore (1977). The descriptions of dialogue games in the literature do not generally distinguish between games that only lay down the rules for how to perform very general communicative functions, and rules stating which sub-projects need to be realised in order to carry out a communicative project.

Inspired by the string theory of events in work by Fernando (2006), Cooper and Ginzburg (2015), and Cooper (2016) describe a conversational game as a type of string of events where each event is (more or less) expected by the agents involved in the realisation of the string. If $T_{1}$ and $T_{2}$ are types of events, then, $T_{1} \frown T_{2}$ is the type of strings of events $a \frown b$ such that $a: T_{1}$ and $b$ : $T_{2}$. 
On this view, it is not only conversational games that are perceived as string types - activity, project and conversational game may all be described as strings of events. On this view the distinction between a conversational game and a communicative project is not structural. Rather, the difference is related to the domain. An activity type is domain specific, that is it involves particular roles which are strongly associated with specific rights, obligations, etc. as well as associated settings and artefacts. A communicative project tends to be less domain specific - for example agreeing on something is a communicative project which may occur in various activity types. However, the carrying out of an activity of a particular kind often also requires specific communicative projects to be carried out.

Conversational games, on the other hand, are more general and reflect conversational practices which are or could be part of most communicative activities, such as asking or replying to questions, backtracking, giving feedback, etc. We define a conversational game as the minimal set of linguistic actions that need to be performed in order to realise a communicative project—similar to "speech act sequence" in van Dijk (1979). Examples of conversational games could be "request game", "suggestion game", "clarification game" and "assertion game”.

\subsection{The Suggestion Game}

A relevant game type in relation to the dialogue in (5.8), is the suggestion game. In this section we will describe this game in terms of informal rules and conditions which state what should be allowed within the game:

- The suggestion game is played by at least two players, though more players are possible.

- The players have a shared project which they intend to carry out together.

- One of the dialogue participants makes a suggestion. For the sake of the game it does not matter which player has made the first move. We refer to whoever has done so as player 1.

- The suggestion by player 1 may optionally be followed by a motivation for the suggestion, again by player 1 .

- Another player (who is then player 2) responds to the suggestion by accepting or rejecting the move. Note that this move does not necessarily have to be an actual response. Depending on the level of grounding we are willing to accept, abstaining from protesting might be enough to signal acceptance of a given suggestion.

We could describe the suggestion game as illustrated in Figure 1. 


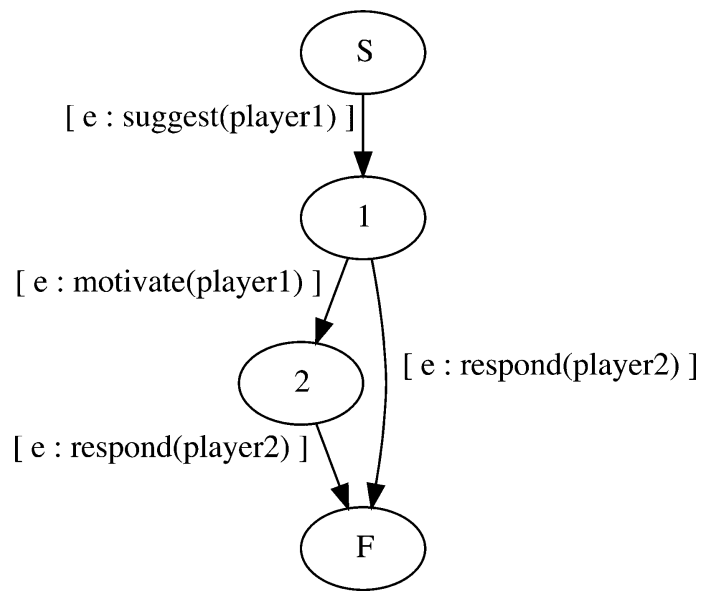

FIGURE 1 The Suggestion game

The rules above would suffice to account for an interaction where Player 1 makes a suggestion $\varphi$ to player 2 , who responds by either accepting or rejecting it. However, a general set of rules that would account for the suggestion game would also have to allow for a less straightforward carrying out of the project "deciding which route to take". There could be questions, for example clarification questions or questions regarding other aspects of the context (time, place etc.) or the suggested route. Another possibility is that player 2 asks for a reason for choosing the suggested route. This would be perfectly acceptable dialogue behaviour, and players must be allowed, within the suggestion game, to move into games of other types like the clarification game or the motivation game.

We see the ability to move between games as a general rule for all conversational games. This reflects the expectations we have when engaging in dialogue-if you ask someone a question, you know that it is likely that you will get a reply. However, we can still account for dialogue behaviour which does not conform to one particular game, since we allow dialogue participants to introduce new games - and even new projects — and we also allow dialogue participants not to play the game.

So, we want rules which allow for the suggestion game to be played in a number of different ways, including detours into other games. But let us leave that aside for the moment, and just consider the possibilities realised in (5.8). If we want to represent this dialogue in terms of updates of information states, we need rules handling not only the explicit moves represented in Figure 1, but also tacit updates of the DGB. Tacit moves within a game represent inferences and other internal processes. We will now have a look at some of the updates of $A$ 's and $B$ 's DGB throughout (5.8). 


\subsection{Analysing a Suggestion Dialogue}

\subsubsection{Initial Tacit Moves}

Since the notion of conversational game is linked to the acceptable moves in a conversation, rather than to the concrete results which conversational participants hope to get out of the interaction (as in the case of a communicative project) we introduce a new field—games — onto the gameboard.

In order to start a conversation, an agent searches her resources for a conversational strategy (a conversational game) to carry out the project. In case of the dialogue in (5.8), where one dialogue participant suggests to another which route they should take to work, a relevant game type is the suggestion game just described in Section 5.4. It is not until the first move is made and the conversational participants thus consider it shared information that this is the game which is being played, that the game appears on the shared game board.

At the beginning of the interaction the DGB of dialogue participant $A$ is empty apart from the field 'project', which we assume to be shared since the necessity of picking a route is obvious to both $A$ and $B$ in the context. As we remember from Chapter 4, a communicative project is a limited task or activity which is being carried out at least to some extent by means of communication. We represent a project as a type of event to be brought about by a number of agents. In (5.10) we see the type of a decision project, $T_{\text {DecisionProject }} A_{1}, \ldots, A_{n}$ are dialogue participants and Issue the thing that is to be decided upon.

(5.10) $T_{\text {DecisionProject }}=\left[\mathrm{e}:\right.$ decide $\left(\left\{A_{1}, \ldots, A_{n}\right\}\right.$, Issue $\left.)\right]$

For the purposes of this dialogue, only one communicative project needs to be carried out. However, by letting the type of project on the DGB be list(RecType), we are also able to model several projects to be carried out one after the other to fulfil some complex goal (linguistic or other). We would also be able to account for projects suddenly appearing in the information states of dialogue participants due to sudden events, such as "find shelter from the rain".

$$
\left[\begin{array}{l}
\text { private }: \text { RecType } \\
\text { shared }:[\operatorname{project}=[[\mathrm{e}: \operatorname{decide}(\{A, B\}, \text { route })]]: \operatorname{list}(\operatorname{RecType})]
\end{array}\right]
$$

(5.11) shows the type of the speaker, $A$ 's, information state at the beginning of the interaction in (5.8). For now we are interested only in the information state of dialogue participant $A$, not that of the listener, dialogue participant $B$. "Route" represents the issue of which route to take, in (5.8). 
The first update of the dialogue gameboard is an update of 'private games', that is the repository of conversational games which are salient with respect to a dialogue participant in a given context. Before we move on to how we want to represent this update in TTR, let us have a look at the nature of projects and games in terms of types.

As illustrated in (5.10) we perceive a project as a record type representing the type of an event where a number of individuals (in this case $A$ and $B$ ), jointly perform some action (in this case making a decision) regarding some non-decided-upon issue. In this case, the issue which is being deliberated is which route to take to work.

We may think of the development of a conversation as a finite state automaton where the arrows leading from one state to another correspond to the linguistic moves of the conversation, as represented in Figure 1. Instead of focusing on the states between the moves, we could focus on the sequence of moves themselves when defining a conversational game. We would then get a string of move types. The type in (5.12) for example, is of strings of moves comprising the type of suggestion game, $T_{\text {SuggestionGame }}$ - - a suggestion by player 1 followed by an optional motivation by player 1 , followed by a response (acceptance or rejection) by player 2 . We represent move types as record types. A game of the type in (5.12) is made up of a suggestion, followed by an optional motivation by the dialogue participant who made the suggestion, followed by a response (either an accept- or a reject move) by the other player. $^{2}$

$$
\begin{aligned}
& T_{\text {SuggestionGame }}=[\mathrm{e}: \text { suggest }(\text { player } 1)] \\
& \quad[\mathrm{e}: \text { respond }(\text { player })]
\end{aligned}
$$

The notation $[\mathrm{e}:$ motivate(player $)]^{\leqslant 1}$ means that the suggestion move is followed by at most one motivation move $\left(^{\leqslant 1}\right)$. One could argue that a suggestion might be followed by more than one move motivating the suggestion, and it would of course be possible to alter $\leqslant 1$ to $\leqslant 2$ or $\leqslant 3$ depending on how many motivation moves the model should allow. The string in (5.12) represents the type of a suggestion game on an abstract level-from this type we learn the

2 In Chapter 4, we represented move types as ptypes constructed from three-place predicates, such as suggest $(x, y$, ctnt), where $x$ is the speaker, $y$ is the addressee and ctnt is the content. In this chapter, we will sometimes use one-place predicates like suggest $(x)$, where $x$ is the speaker, to represent a slightly more abstract move type (where addressee and content are left unspecified). We will also use yet more abstract move types like Suggest which do not specify any arguments. One may think of these types as related in a subtype hierarchy, so that for example suggest $(x, y, c$ tnt $) \sqsubseteq \operatorname{suggest}(x) \sqsubseteq$ Suggest. 
sequence of move types involved and the relation between the roles that are necessary to play the game. However, in order for the game to work as a motor in the dialogue driving the updates, we need to assign the roles of the game to the individuals present in the context. For example, the player who initiates the game by making a suggestion has to be distinct from the player who acknowledges that suggestion.

\subsubsection{Rules for Updating Private Games}

There are at least two different scenarios which would lead to an update of private games. First, there is the type of situation where the presence of a project on the DGB causes an agent to search his long term memory for a strategy by which to carry out that project, and load it onto the DGB. The second is when there is already a game on private games that would suffice to carry out the project. Assume for example that $A$ has been thinking since he got out of bed in the morning that he wants to pass Walnut Street on his way to work. He has been meaning to suggest it for a while (or maybe hoping that $B$ will suggest it), thus the suggestion game is activated on his private DG B. When $A$ and $B$ reach a junction the issue of which route to take becomes necessary to address, and the project appears on the shared DGB. In this case the only update necessary on $A$ 's DG B is to place $T_{\text {SuggestionGame }}$ first in the list of games, while $B$ has to retrieve the game from long term memory and load it onto private games. As mentioned in the previous chapter, the idea is that the update rules are combined with a control algorithm selecting which rule to apply in a given context. In Figure (2) we see a visualisation of the algorithm controlling the update of private games.

\subsubsection{Update Private Games}

We want the first rule $f_{\text {ud_pr_aames }}$ to apply in a context where an agent has a project on her gameboard, but the game first on the list of private games is not relevant to carry out the project. The agent is then licensed to either reraise a game already on private games (but not first on the list) or to load a relevant game from resources onto private games. Now, one question that arises here is what it means to be a relevant game in relation to a particular project. One way of describing this would be in terms of licences in an agent's resources. If an agent has in her resources a link between a type of project $T_{P}$ and a type of game $T_{G}$, she has a licence to carry out a communicative project of type $T_{P}$ by means of $T_{G}$, and may load it onto 'private.games' on her DGB.

Which types of games are relevant to carry out particular types of projects is an empirical question. We think of the update rules licensing the carrying out of a project by means of a particular type of game as reflecting the pragmatic norms of a community. One way of modelling how an agent selects a 


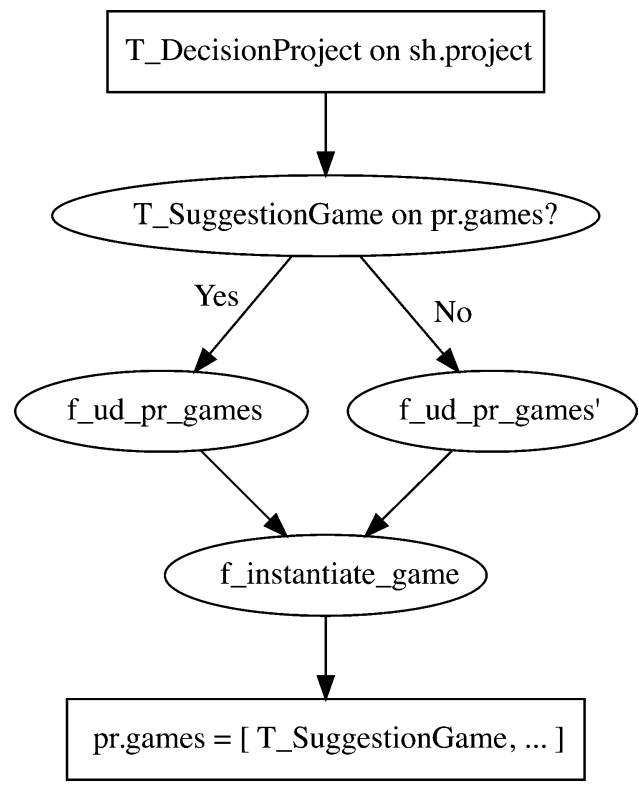

FIGURE 2 Update of private games

strategy - for example choosing between an indirect and a direct speech actwould be to extend the model with a probabilistic component (see for example Eshghi and Lemon (2014)). However, in the limited model we are focusing on here, we assume that we have access to only one type of game which is relevant to the project at hand. Moreover, it seems to us that a limited set of project types and game types would suffice to account for a large number of dialogue situations. Thus, for each project type we would introduce a set of postulates defining which games could be relevant to carry out a project of that type. We use the notation "relevant_to $\left(T_{1}, T_{2}\right)$ " to represent relevance of $T_{1}$ in relation to $T_{2}$.

When a communicative project appears on an agent's DGB and the agent initiates carrying out the project there are, as mentioned above, two possibilities. Either there is a game present in the private games field of the DG B by means of which the project can be carried out, or there is not. In the first case we want to make sure that the appropriate game is moved up to the first slot on the list of private games. In the second case, we want to pick an appropriate game from the agent's long term memory, and place it first on the list of private games. The update of 'private.games' thus consists of three rules: $f_{\text {ud_pr_ames }}$ for reraising a game, $f_{\text {ud_pr_games }}$ for uploading a game from resources, and-to complete the update $-f_{\text {inst_game. }}$ In an instantiated game the roles (player1, player2, etc.) are assigned to dialogue participants from the point of view of the participant on whose gameboard the instantiated game appears. This means in the case of the 
suggestion game, that when $A$ starts carrying out a decision making project by initiating a game of type $T_{\text {SuggestionGame, }}$ she has also taken on the role of 'player 1 ' in that game. In every move type of the instantiated game on her DGB the move to be carried out by player 1 will be assigned to SELF, the ones by player 2 to OTHER.

Even though instantiated games involve assignments of roles to dialogue participants, we still want to be able to treat them as types. For this reason, the type of games is a join type. A join type is a disjunction such that, for any two types $T_{1}$ and $T_{2}$ you can form the join $T_{1} \vee T_{2}$. a : $T_{1} \vee T_{2}$ just in case either a : $T_{1}$ or a $: T_{2}$ (Cooper and Ginzburg, 2012). This means that the type of games, $T_{\text {Game, }}$ in our theory is a join of the types non-instantiated game, $T_{\text {NonnsstGame }}$ and instantiated game, $T_{\text {InstGame }}$ as defined in (5.13):

(5.13) $a: T_{\text {Game }}$ iff $a: T_{\text {NonInstGame }}$ or $a: T_{\text {InstGame }}$

By defining the type of game as a join, we make sure that we can handle situations where, for example, something sudden and unexpected happens, and dialogue participant needs to postpone the initiation of a game already on the DGB. We will look at the instantiation process in more detail further on in this section.

$$
\begin{aligned}
& f_{\text {ud_pr_games }}= \\
& \lambda r:\left[\begin{array}{l}
\text { pr: }:\left[\text { games }: \operatorname{list}\left(T_{\text {Game }}\right)\right] \\
\text { sh }:\left[\operatorname{project}=\left[T_{\text {DecisionProject }}\right]: \operatorname{list}(\text { RecType })\right]
\end{array}\right] \cdot \lambda e:\left[\begin{array}{l}
\mathrm{g}: T_{\text {SuggestionGame }} \\
\mathrm{c}_{1}: \text { in }(\mathrm{g}, r . \text { pr.games })
\end{array}\right] \\
& \quad\left[\mathrm{pr}:\left[\text { games }=[\mu(e . \mathrm{g}, r \text {.pr.games })]: \operatorname{list}\left(T_{\text {Game }}\right)\right]\right]
\end{aligned}
$$

In (5.14), $f_{\text {ud_pr_games }}$ takes a situation of the type where there is a decision project on 'shared.project' and, if there is a game of type $T_{\text {SuggestionGame }}$ on private games in that record, the function returns a type of situation where that game type is first on 'private.games'. (See (4.2.3) on the function of $\mu$.)

We think of the update rule $f_{\text {ud_pr_games' }}$, as seen in (5.15) as a function from an information state where an agent has a decision project on her gameboard but no game of type $T_{\text {SuggestionGame }}$ on the list of games on 'private.games', 3 to an information state where the agent has a decision project on 'shared.project' and a suggestion game first on 'private.games'. In this case the game $T_{\text {SuggestionGame }}$ has to be retrieved from parts of the agent's resources which are external to the DGB.

3 There may be other games on the list of private games, just not the game suggestion game. 
(5.15) $f_{\text {ud_pr_games' }}=$

$\lambda r:\left[\begin{array}{l}\mathrm{pr}:\left[\text { games }: \operatorname{list}\left(T_{\text {Game }}\right)\right] \\ \mathrm{sh}:\left[\text { project }=\left[T_{\text {DecisionProject }}\right]: \operatorname{list}(\text { RecType })\right]\end{array}\right] \cdot \lambda e:\left[\begin{array}{l}\mathrm{g}: T_{\text {SuggestionGame }} \\ \mathrm{c}_{1}: \neg \text { in }(\mathrm{g}, r \text {.pr.games })\end{array}\right]$

$\left[\mathrm{pr}:\left[\right.\right.$ games $=[e . \mathrm{g} \mid r$.pr.games $\left.\left.]: \operatorname{list}\left(T_{\text {Game }}\right)\right]\right]$

The functions in (5.14) and (5.15) are similar to the update functions discussed by Cooper (2016, pp. 24-26). In order to obtain the required update of such a function we need to apply it to the current information state - that is the information state at the start of the update-of the agent whose information state we seek to capture. Let us consider a scenario where agent $A$ has previously considered suggesting Walnut Street, but was distracted by an event which the agent has just observed. This caused another conversational game, $T_{G_{X^{\prime}}}$ to appear on the DGB. His initial information state is thus of the type in (5.16), which we refer to as $T_{\text {current }}$.
a. $T_{\text {current }}=\left[\begin{array}{l}\operatorname{pr}:\left[\text { games }=\left[T_{G_{X}}, T_{\text {SuggestionGame }}\right]: \operatorname{list}\left(T_{\text {Game }}\right)\right] \\ \text { sh }:\left[\text { project }=\left[T_{\text {DecisionProject }}\right]: \operatorname{list}(\text { RecType })\right]\end{array}\right]$
b. $s_{\text {current }}: T_{\text {current }}$

Before we apply the function we need to make sure that the type of the current information state is a subtype of the domain type of $f_{\text {ud_pr_games }}$. We should point out here that the type of the current information state might very well have other fields such as a shared game, a latest utterance, shared beliefs, etc., and still be a subtype of the domain type of $f_{\text {ud_pr_games }}$.

In (5.17) we see the application of $f_{\text {ud_pr_games }}$ to $s_{\text {current }}$, followed by an asymmetric merge (see Section 4.1.1.1) of the result of that function application and the type $T_{\text {current }}$ of $s_{\text {current }}$ (as well as $e_{1}$ witnessing the condition that $T_{\text {SuggestionGame }}$ is in $s_{\text {current }}$ pr.games).

$$
\begin{aligned}
& \text { a. } f_{\text {ud_pr_games }}\left(s_{\text {current }}\right)\left(e_{1}\right)= \\
& {\left[\mathrm{pr}:\left[\text { games }=\left[T_{\text {SuggestionGame }}, T_{G_{X}}\right]: \operatorname{list}\left(T_{\text {Game }}\right)\right]\right]} \\
& \text { b. } T_{\text {current }} \wedge\left[\mathrm{pr}:\left[\text { games }=\left[T_{\text {SuggestionGame }}, T_{G_{X}}\right]: \operatorname{list}\left(T_{\text {Game }}\right)\right]\right]= \\
& {\left[\begin{array}{l}
\text { pr }:\left[\text { games }=\left[T_{\text {SuggestionGame }}, T_{G_{X}}\right]: \operatorname{list}\left(T_{\text {Game }}\right)\right] \\
\text { sh }:\left[\operatorname{project}=\left[\left[\mathrm{e}: \operatorname{decide}\left(\left\{A_{1}, A_{2}\right\}, \text { Issue }\right)\right]\right]: \operatorname{list}(\text { RecType })\right]
\end{array}\right]}
\end{aligned}
$$

\subsubsection{Instantiation of Game}

After an update putting a game which is a subtype of $T_{\text {SuggestionGame }}$ first on the list of private games (either by $f_{\text {ud_pr_games }}$ or $f_{\text {ud_pr_games' }}$ ), we need to instantiate the game, that is associate the roles of the game with the players in this particu- 
lar situation. To do this we apply the function $f_{\text {inst_- } T_{\text {suggestion } C a m e}}$ to a record assigning the values 'SELF' and 'OTHER' to the roles of the suggestion game.

(5.18) $f_{\text {inst_ } T_{\text {Suggestioncame }}}=$

$$
\begin{gathered}
\lambda r:\left[\begin{array}{l}
\text { player1: Ind } \\
\text { player2 }: \text { Ind }
\end{array}\right] \cdot[\mathrm{e}: \operatorname{suggest}(r \text {.player })] \frown[\mathrm{e}: \text { motivate }(r \text {.player })]^{\leqslant 1} \\
{[\mathrm{e}: \operatorname{respond}(r \text {.player } 2)]}
\end{gathered}
$$

For dialogue participant $A$ in our current example this assignment would be that in (5.19).

(5.19) $\quad r=\left[\begin{array}{l}\text { player1 }=\text { SELF } \\ \text { player2 }=\text { OTHER }\end{array}\right]$

In (5.20) we see the application of $f_{\text {inst_ } T_{\text {Suggestion Came }}}$ to $r$.

$$
\begin{aligned}
& f_{\text {inst_ } T_{\text {Suggestioncame }}}(r)= \\
& {\left[\text { e }: \operatorname{suggest}\left(\left[\begin{array}{l}
\text { player1 }=\text { SELF } \\
\text { player2 }=\text { OTH ER R }
\end{array}\right] \text { player1 }\right)\right] \frown} \\
& {\left[\mathrm{e}: \operatorname{motivate}\left(\left[\begin{array}{l}
\text { player1 }=\text { SELF } \\
\text { player2 }=\text { OTHE R }
\end{array}\right] \cdot \text { player1 }\right)\right] \leqslant 1 \frown} \\
& {\left[\mathrm{e}: \operatorname{respond}\left(\left[\begin{array}{l}
\text { player1 }=\text { SELF } \\
\text { player2 }=\text { OTHER }
\end{array}\right] \cdot \text { player2 }\right)\right]=} \\
& {[\mathrm{e}: \operatorname{suggest}(\mathrm{SELF})]^{-}[\mathrm{e}: \text { motivate(SELF) }]^{\leqslant 1} \frown} \\
& \text { [e : respond(OTHER) }
\end{aligned}
$$

The instantiated suggestion game would in this situation thus be $T_{\text {SuggestionGame- }}$ Inst as seen in (5.21):

(5.21) $T_{\text {SuggestionGameInst }}=[\mathrm{e}: \operatorname{suggest}(\mathrm{SELF})] \frown[\mathrm{e}: \operatorname{motivate}(\mathrm{SELF})] \leqslant 1 \frown$

$$
\text { [e : respond(OTHER })]
$$

\subsubsection{Updating the Agenda}

An important aspect of the notion of conversational game is that players (conversational participants), by identifying an utterance as being part of a particular game, get an idea of which moves are likely to follow and what part they should expect to play over the next few turns of the dialogue. In this sense conversational games may be seen as engines driving dialogues forward. Once a game is loaded onto the gameboard and roles are assigned to individuals in the context, an agent involved in a conversation can at any stage of the game look 
at her gameboard and know what options are available if she wants to keep playing the game. Before the update of the agenda, agent $A$-if playing the suggestion game - has on her private games the instantiated game $T_{\text {SuggestionGameInst }}$ which we see in (5.22).

Now, we want an update rule that would load the first available move of the game which is to be carried out by SELF, onto the agenda. We have a set of rules pertaining to the suggestion game that governs the dynamics of the agenda, which is inherent in the suggestion game in (5.21). This set of rules is similar to that used to update the agenda in Cooper (2016). However, in Cooper's approach, there are rules pushing moves onto the agenda which are to be made by agents other than 'SELF'. Since we have a conversational game specifying what we expect of others and ourselves in the employment of that particular game, we have chosen to use the agenda only for moves that are to be made by the agent whose agenda they are on. The agenda is part of the 'private'-field of an agent's gameboard, and is represented as a record type (move type). Each move type has a label 'e' paired with one of a set of speech act types like Suggest, Ask, Assert, etc. There are a number of constraints on such move types having to do with the roles of the agents involved in dialogue, $\mathrm{c}_{\text {actor }}$. There could also be more constraints. Further, there is a label 'ctnt' for content, which-after the first update of the agenda - will not yet be associated with a specified content.

The first rule to be employed of the rules of the suggestion game is a "starting rule" in (5.22), stating that if a player has an empty agenda and a suggestion game on his private gameboard, he may, within the suggestion game, push a suggestion onto the agenda. We refer to this rule as $f_{\text {update_agenda_suggestion. }}$.

$$
\begin{aligned}
& f_{\text {update_agenda_suggestion }}= \\
& \lambda r:\left[\operatorname{pr}:\left[\begin{array}{l}
\text { agenda }=[]: \operatorname{list}(\text { RecType }) \\
\text { games }=\left[T_{\text {SuggestionGamernst }}\right]: \operatorname{list}\left(T_{\text {Game }}\right)
\end{array}\right] .\right.
\end{aligned}
$$

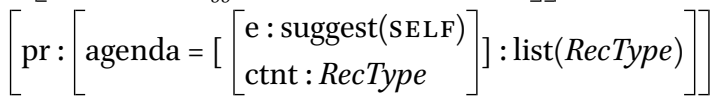

The content of the move type that ends up on the agenda is unspecified. $f_{\text {update_agenda_suggestion }}$ is applied to a record of the type in (5.23):

$$
\left[\begin{array}{l}
\left.\left.\operatorname{pr}:\left[\begin{array}{l}
\text { agenda }=[]: \operatorname{list}(\text { RecType }) \\
\text { games }=\left[T_{\text {SuggestionGameInst }}\right]: \operatorname{list}\left(T_{\text {Game }}\right)
\end{array}\right]\right]: \operatorname{list}(\text { RecType })\right] \\
\operatorname{sh}:[\operatorname{project}=[[\mathrm{e}: \operatorname{decide}(\{A, B\}, \text { route })]
\end{array}\right.
$$


We apply the function in (5.22) to the current information state of the type in (5.23), and asymmetrically merge the current state type with the result of function application. In (5.24) we see the type of $A$ 's information state after the rule has been applied.

$$
\left[\begin{array}{l}
\operatorname{pr}:\left[\begin{array}{l}
\text { agenda } \left.=\left[\begin{array}{l}
\mathrm{e}: \operatorname{suggest}(\mathrm{SELF}) \\
\mathrm{ctnt}: \text { RecType }
\end{array}\right]\right]: \operatorname{list}(\text { RecType }) \\
\text { games } \left.=\left[T_{\text {SuggestionGamelnst }}\right]: \operatorname{list}\left(T_{\text {Game }}\right)\right)
\end{array}\right] \\
\operatorname{sh}:[\operatorname{project}=[[\mathrm{e}: \operatorname{decide}(\{A, B\}, \operatorname{route})]]: \operatorname{list}(\text { RecType })]
\end{array}\right]
$$

The next update rule provided by the conversational game (although this rule is actually general and applicable to any conversational game) is a rule saying that if we have an item on the agenda which is to be performed by SELF and whose content is specified, that is, the label 'ctnt' has one specific value ([ctnt=T:RecType]), then the agent is allowed to make that move and push the next move onto the agenda (we will get back to this rule soon). However, at the moment the item on the agenda is not specified in terms of content-the label is just typed RecType (ctnt:RecType). In order to add a content specific move to the agenda, the agent needs to search her resources for relevant facts and ways of reasoning about the situation and the project at hand.

\subsubsection{Drawing on Topoi to Specify Move Content}

So far in this chapter we have considered mechanisms of things like speech act sequences and turn taking. We will now move on to consider how a dialogue participant may reason given the topoi which are available to him or her in relation to a particular goal.

For example, let us assume that an agent is involved in a conversational game according to which he is expected to make a suggestion regarding some future action. One way for the agent to decide which suggestion to make, is to reason based on the relevant topoi available to him.

There is evidence that the strong assumptions of intentionality associated with, for example, Gricean pragmatics and Relevance Theory, sometimes do not hold (Gregoromichelaki et al., 2011). Thus, conversational participants do not necessarily reason about how their moves will be interpreted. Rather, reasons for making specific claims, suggestions and other moves in conversation are not always arrived at through conscious reasoning. In such cases a motivation or reason for the content of a claim or suggestion may be added post hoc, if the agent is asked to motivate his actions.

However, there are contexts where claims or suggestions are arrived at after planning involving conscious reasoning. This is important not least in the con- 
text of modelling a dialogue system. A dialogue system which has identified that it is supposed to make the first move in a suggestion game requires a structured way of choosing how this move should be specified. That is, which content it should have, based on the available possibilities. The suggestion made should be based on reasoning that may also be accounted for. Thus, at this point in the dialogue in (5.4), we assume that the agent is about to make a suggestion based on its role in the current interaction. However, we assume that the precise content of this suggestion is not yet specified.

\subsubsection{Integrate Private Resource Topos and Belief}

In order to be able to specify the content of the agenda, the agent must access relevant information. The update we are looking for is an update of private topoi and beliefs. This update rule should add information to the agent's private beliefs and topoi which is relevant to the project at hand. The relevance of the project is slightly different here than for adding a private game to the gameboard. In the latter case the relation between the project "decide(which route to choose)" and the suggestion game clearly has more to do with the fact that a decision is to be made than with what the decision concerns. In contrast, in the case of topoi and relevant beliefs it seems more likely that the choice of topos is more domain specific. The simplest way of doing this is to have a rule saying that if you have an information state that includes a particular project $P$ and an unspecified item on the agenda, then you can load a particular set of topoi and beliefs onto the gameboard.

Before we move on to present a more detailed account of this update rule, let us consider $\tau_{\text {shorter }}$-representing a topos saying that if one route is shorter than another, take that route. As in previous chapters we represent topoi and enthymemes as functions from records to record types. The topos which is loaded onto the 'private' field of $A$ 's gameboard should capture the notion that if we have a choice between a shorter route and a longer route, we take the shorter one.

This rule of thumb is not absolute-a shorter route might take much longer because of roadworks, or be associated with other unappealing features and therefore not preferable. The agents involved in the situation might also for some reason prefer a longer walk. In many cases however, the rule of thumb that we should choose shorter rather than longer routes applies. Informally, we can say that the domain type includes two routes of which one is shorter than the other and one agent (or set of agents) who has to make a choice between two routes of which one is shorter. The result type is the type of situation where the agents walk along the shorter route. In (5.25) we see a suggestion of what such a topos might look like. 


$$
\left.\begin{array}{l}
\text { (5.25) } \tau_{\text {shorter }}= \\
\left.\qquad \begin{array}{l}
\mathrm{x}: \text { Ind } \\
\mathrm{y}: \text { Ind } \\
\mathrm{z}: \text { Ind } \\
\mathrm{c}_{\text {agent }}: \operatorname{agent}(\mathrm{z}) \\
\mathrm{c}_{\text {route }}: \operatorname{route}(\mathrm{x}) \\
\mathrm{c}_{\text {route }}: \operatorname{route}(\mathrm{y}) \\
\mathrm{c}_{\text {shorter_than }}: \operatorname{shorter} \text { than }(\mathrm{x}, \mathrm{y}) \\
\mathrm{c}_{\text {choose_between }}: \text { is_choosing_between }(\mathrm{z}, \mathrm{x}, \mathrm{y})
\end{array}\right] \cdot[\mathrm{e}: \operatorname{take}(r . \mathrm{z}, r . \mathrm{x})]
\end{array}\right]
$$

We think of beliefs in an agent's long term resources as assumptions about the world including, but not limited to, facts. We model beliefs as record types. The relevant belief in this case would be that Walnut Street is shorter than the other possible route-let us call it Maple Street. We refer to this belief, represented by the record type in $(5.26)$, as $T_{b W S t \_s}$.

(5.26) $T_{b W S t \_s}:\left[\begin{array}{l}\mathrm{x}=\text { Walnut Street }: \text { Ind } \\ \mathrm{y}=\text { Maple Street }: \text { Ind } \\ \mathrm{c}_{\text {shorter_than }}: \text { shorter_than }(\mathrm{x}, \mathrm{y})\end{array}\right]$

Let us now look at the update rule for integrating topoi and beliefs on the private DGB. We want the rule to apply when an agent has a move type on the agenda which is not specified for content. The rule should capture what is a context relevant way of reasoning. At this point the content of the item on the agenda is not fully specified. Thus there is information about the function of the move that is to be made (to make a suggestion) while we do not know anything about the content. The label 'ctnt' in the move type on the agenda is therefore associated with the type ERec, whose only witness (the only thing that is of that type) is the empty record. For a definition of the empty record, see Cooper (2016, p. 47).

In $(5.27)$ we see the update function $f_{\text {integrate_pr_resource_topos }}$. 


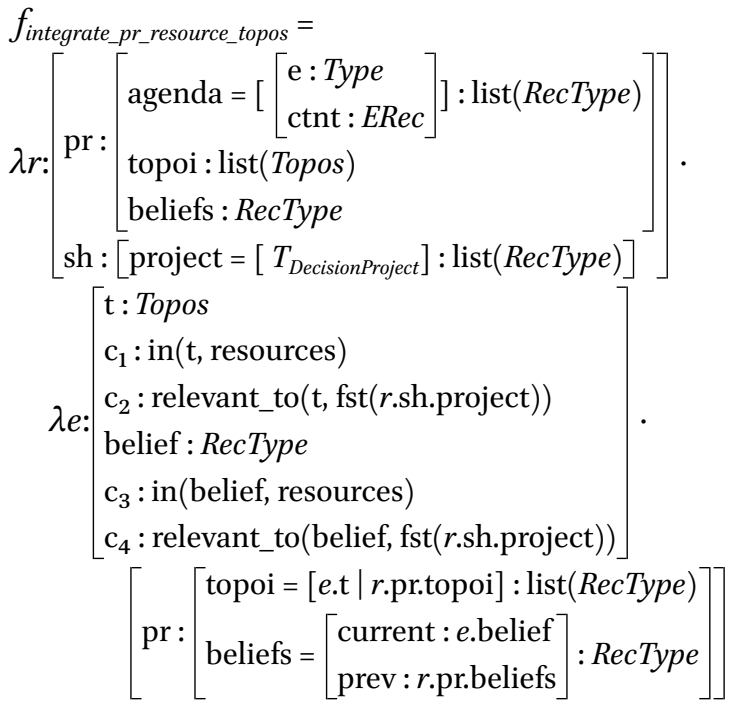

The rule above in (5.27) says that if you have a move type on the agenda whose content is of type ERec, and there is a topos in your resources such that it relevant to the project at hand (the first item on the list of projects, or maxproject following max-QUD in Ginzburg's terminology) and a belief such that it is relevant to the project at hand, then you are allowed to integrate that topos and that belief on your private DGB. We have ignored the possibility that the integration of beliefs and topos might not be simultaneous and that just one of them could be enough to underpin the specification of content of the next move. For example, there might be cases where a topos alone is sufficient to sanction a specification of the agenda. This would be the case for example if one agent makes a suggestion and the other agent accepts, believing that people usually have a good reason for making a suggestion. This could possibly lead to the other agent accepting without having taken any additional information into account.

Though we are aiming at modelling the kind of updates that would allow an agent to participate in a particular type of dialogue by accounting for the kind of dialogue behaviour present in such a situation, we do not claim that our model necessarily mirrors the cognitive procecesses that actually take place. It seems likely that the topoi involved and other beliefs interact rather than one of them preceding the other. Thus we choose to represent both of these updates in one rule. However, it would be possible to turn them into two separate rules if one would wish to represent a dependency between beliefs and topoi where the integration of one precedes the integration of the other. 


\subsubsection{Integrate Private Topos}

In the previous chapter, Section (4.2.1), we considered the integration of shared topoi on the DGB. We suggested a pair of rules where one looks for relevant topoi already on shared topoi (but not at the top of the list), and moves them to the top of the list. If there is no such topos already on shared topoi, another function looks in private topoi for a relevant topos and-if there is such a topos-loads it onto shared topoi. In the situation we are currently considering, we might want to add a similar rule. This rule would search private topoi for a relevant topos and-if such a topos is present-place it first on the list of private topoi, using the function $\mu$ as described in Section 4.2.3.

$$
\begin{aligned}
& \begin{array}{l}
f_{\text {integrate_pr_topos }}= \\
\lambda r:\left[\begin{array}{l}
\operatorname{pr}:\left[\begin{array}{l}
\text { agenda } \left.=\left[\begin{array}{l}
\mathrm{e}: \text { Type } \\
\text { ctnt }=\text { ERec }: \text { RecType }
\end{array}\right]\right]: \operatorname{list}(\text { RecType }) \\
\text { topoi }: \operatorname{list}(\text { Topos })
\end{array}\right] \\
\text { sh }:[\text { project }=[[\mathrm{e}: \operatorname{decide}(\{A, B\}, \text { route })]]: \operatorname{list}(\text { RecType })]
\end{array}\right] .
\end{array} \\
& \lambda e:\left[\begin{array}{l}
\mathrm{t}: \text { Topos } \\
\mathrm{c}_{1}: \text { in }(\mathrm{t}, r \text {.pr.topoi }) \\
\mathrm{c}_{2}: \text { relevant_to }(\mathrm{t}, \text { fst }(r \text {.sh.project }))
\end{array}\right] \text {. } \\
& {[\mathrm{pr}:[\text { topoi }=\mu(\text { e.t, } r \text {.pr.topoi }): \operatorname{list}(\text { RecType })]]}
\end{aligned}
$$

\subsubsection{Specify Content of Suggestion on Agenda}

We have one more silent move left to account for before we reach the point where a linguistic move is actually made, and that is an update of the agenda where information in the dialogue participant's private depository of salient topoi and beliefs is used to add content to the move type first on the agenda. Unlike the update rules for private games and private topoi and beliefs, the rule for specifying the agenda is not about loading information from an agent's resources onto her gameboard, but about assembling information already on the gameboard, turning it into a content-specific type and pushing that type onto the agenda.

We do not present any theory of how the information on the gameboard is turned into actual utterances. We will just say that the information on topoi and beliefs affect the content of the utterance. For example, in a case where a route is to be suggested, a topos regarding routes, such as $\tau_{\text {shorter }}$ combined with beliefs about the available routes can be combined to contribute content to the suggestion move. The most recently added items on topoi and beliefs are relevant to the move the agent is about to make.

The update function would thus be a function from a record of a type where there is a project, a game, a topos and a set of beliefs-but no specified content 
on the agenda - to a type of information state where there is a move type on the agenda where the label 'ctnt' is associated with a specific content. This rule is represented below in (5.29):

$$
\begin{aligned}
& f_{\text {specify_suggestion_content }}= \\
& \lambda r:\left[\begin{array}{l}
\operatorname{pr}:\left[\begin{array}{l}
\text { agenda } \left.=\left[\begin{array}{l}
\text { e }: \text { Suggest } \\
\text { ctnt }=\text { ERec }: \text { RecType }
\end{array}\right]\right]: \operatorname{list}(\text { RecType }) \\
\text { topoi }: \operatorname{list}(\text { Topos }) \\
\text { beliefs }: \operatorname{list}(\text { RecType })
\end{array}\right] \\
\text { sh : }[\text { project }: \operatorname{list}(\text { RecType })]
\end{array}\right] . \\
& \lambda e:\left[\begin{array}{l}
\mathrm{t}: \text { Topos } \\
\mathrm{c}_{1}: \text { in }(\mathrm{t}, r \text {.pr.topoi }) \\
\mathrm{c}_{2}: \text { relevant_to }(\mathrm{t}, \text { fst }(r \text {.sh.project })) \\
\text { belief }: \text { RecType } \\
\mathrm{c}_{3}: \text { in }(\text { belief, } r \text {.pr.beliefs }) \\
\mathrm{c}_{4}: \text { relevant_to }(\text { belief, fst }(r . \text { sh.project })) \\
\text { ctnt }: \text { RecType } \\
\mathrm{c}_{5}: \text { derived_from }(\mathrm{ctnt},\{\mathrm{t}, \text { belief }\})
\end{array}\right] . \\
& {\left[\operatorname{pr}:\left[\text { agenda }=\left[\left[\begin{array}{l}
\text { e : Suggest } \\
\text { ctnt }=\text { e.ctnt }: \text { RecType }
\end{array}\right]\right]: \operatorname{list}(\text { RecType })\right]\right]}
\end{aligned}
$$

\subsubsection{Identify Suggestion Game}

Based on the latest utterance made, dialogue participant $B$ is able to identify in which conversational game she is being invited to play. Also, dialogue participant $A$ must now consider the game that was up until now private, as explicitly shared. This update rule is specific to the speech act type Suggest. If the latest utterance is a suggestion, then we are allowed to load the suggestion game onto the DGB. We see the rule $f_{\text {identify_suggestion_game }}$ in $(5 \cdot 30)$ :

$$
\begin{aligned}
& f_{\text {identify_suggestion_game }}= \\
& \lambda r:\left[\operatorname{sh}:\left[\begin{array}{l}
\text { l-m }:\left[\begin{array}{l}
\text { prev }: \text { RecType } \\
\text { e }: \text { Suggest }
\end{array}\right. \\
\text { games }: \operatorname{list}\left(T_{\text {Game }}\right)
\end{array}\right]\right] \text {. } \\
& {\left[\text { sh }:\left[\text { games }=\left[T_{\text {SuggestionGame }} \mid r \cdot \text { sh.games }\right]: \operatorname{list}\left(T_{\text {Game }}\right)\right]\right]}
\end{aligned}
$$

When the suggestion game is pushed onto B's shared game, it also has to be instantiated. This is done as in (5.18), but with the assignments adjusted so that the player who has the assignment OTHE R on $A$ 'S DGB has the assignment SELF, and vice versa. 


$$
\left[\begin{array}{l}
\text { player1 }=\text { OT HER } \\
\text { player2 }=\text { SELF }
\end{array}\right]
$$

We have now considered some ways in which the notion of conversational game can be employed to account for updates necessary for actively participating in a conversation. In the following section we will suggest how games can also be useful in the process of accommodating enthymematic relations, that is, assigning a rhetorical relation between two dialogue moves where one is an antecedent and the other a consequent of a enthymematic argument.

\subsubsection{Accommodation of Enthymemes}

The participants in (5.8) are faced with a situation which is common in dialogue - that the rhetorical relation is not made explicit by means of a word like "therefore", "since" or "because". The relation between "Let's take Walnut Street" and "It's shorter" must thus be inferred by dialogue participant $B$. We refer to this as accommodating the enthymeme, parallel to the accommodation of topoi discussed in Chapter 4.

However, in the case of enthymeme accommodation it is the enthymematic relation that is accommodated rather than any semantic content. The question is: On what does dialogue participant $B$ base the accommodation of the enthymeme? In fact, it is probably the case that many factors contribute to the accommodation of the EUD. In the Walnut Street example, (5.8), we have a lexically encoded imperative or exhortation that clearly indicates that we are dealing with a suggestion. Thus, the conversational game which we can expect to be on the shared DGB for all dialogue participants, at this point involves the possibility of a move of type assertion to follow, motivating the content of the suggestion move. Therefore, even though (5.8) does not include a term explicitly signaling that an enthymeme is under discussion, knowledge of basic conversational practice would give this away. However, it seems to also depend on the identification of some topos which supports this interpretation. Intuitively, the rule in (5.32) applies to a situation of a type where an assertion has been made preceded by a suggestion. 
(5.32) $f_{\text {accommmodate_enthymeme }}=$

$$
\begin{aligned}
& \left.\lambda r:\left[\begin{array}{l}
\operatorname{pr}:[\text { topoi }: \operatorname{list}(\text { Topos })] \\
\operatorname{sh}:\left[\begin{array}{l}
\text { prev }:\left[\begin{array}{l}
\text { ctnt }: \text { RecType } \\
\text { e }: \text { Suggest }
\end{array}\right. \\
\text { ctnt }: \text { RecType } \\
\mathrm{e}: \text { Assert }
\end{array}\right]
\end{array}\right]\right] . \\
& \lambda e:\left[\begin{array}{l}
\mathrm{t}: \text { Topos } \\
\mathrm{c}_{1}: \operatorname{in}(\mathrm{t}, r \cdot \text { pr.topoi }) \\
\mathrm{c}_{2}: \operatorname{spec}\left(\mathrm{t}, \lambda r^{\prime}: r . \text { sh.l-m.ctnt } \cdot r \text {.sh.l-m.prev.ctnt }\right)
\end{array}\right] \text {. } \\
& {\left[\mathrm{sh}:\left[\text { eud }=\left[\lambda r^{\prime}: r . \mathrm{l}-\mathrm{m} . \mathrm{ctnt} \cdot r \cdot \mathrm{sh} . \mathrm{l}-\mathrm{m} . \mathrm{prev} . \mathrm{ctnt}\right]: \operatorname{list}(\text { Enthymeme })\right]\right]}
\end{aligned}
$$

\subsection{Summary}

In this chapter we have discussed the issue of how enthymemes may contribute to lower the cognitive load of dialogue participants. Rather than stating, suggesting or requesting something without providing a reason, a dialogue participant can add an utterance giving a reason for the state of affairs described in the previous utterance. Such supporting utterances have been shown to often not convey any new factual information, that is, they are informationally redundant. However, Walker (1996) shows that they still—despite adding linguistic material-make the processing of the entire utterance quicker than if they had been withheld.

We have argued that IRU s can be linked to enthymemes, and that they facilitate the accommodation of a salient topos that could underpin the enthymeme. This could be particularly important in contexts where one agent tries to persuade another not of what is the state of the world, but of changing the world in some way, that is, of doing something. Basing a suggestion on a topos enables an agent to justify it directly by supplying the premise of an enthymematic argument where the suggestion is the consequent.

We suggest some update rules in which conversational games related to speech act type may govern which kind of moves can be expected in a conversation instantiating a particular game type. We focused on the silent updates necessary for a dialogue participant to participate in a dialogue and make moves whose speech act type and order depends on which conversational game the agent is participating in, and whose content depends among other things on the topoi the agent has access to. 


\subsubsection{Enthymemes and Artificial Intelligence}

If IRU s and enthymemes are indeed linked in the way we have argued above, this sheds some light on why enthymemes are such an important part of rhetorical discourse - they simply make it easier to accept the beliefs the speaker wants the audience to encompass or the actions she wants the audience to perform.

Taking rhetoric into account in artificial intelligence (AI) has proven successful in some cases, for example, Miller (2003) reports that rhetorical ethos is central for creating an agent capable of passing the Turing test, that is, not being recognised as a machine by a human user for a certain period of time. Andrews et al. (2006) show that social cues and emotion (pathos) contribute to more human like (and thus more user friendly) dialogue systems.

As the work by Walker (1996) suggests, IRU s can facilitate the processing of the linguistic contributions of an artificial agent by a human. Integrating these insights with a formal theory of enthymematic reasoning provides us with a theory telling us which IRU s are actually helpful in a particular context-the ones supported by salient topoi. This may be particularly useful in contexts where the user of for example a spoken dialogue system is under heavy cognitive load. One such example is in-vehicle dialogue systems.

In a data collection carried out within the DICO project ${ }^{4}$ (Villing, 2009) to test how in-vehicle conversation adapts to shifting cognitive load, Breitholtz and Villing (2008) report many examples of enthymematic arguments, most of which also involve IRU s. The data consist of dyadic in-vehicle interactions where one subject - the passenger - is given a map and a list of destinations and is asked to provide the other subject-the driver-with driving instructions. Both subjects have access to the same situational context, such as street view, traffic intensity, etc. Despite this the passenger often justifies instructions by supplying information about the state of the traffic and street ahead.

For example, in (5.33) it is clear to the driver as well as the passenger that the street they are driving on is ending. By supplying the premise $(5 \cdot 33 \mathrm{~b})$ the passenger points to an enthymeme based on a number of premises, most of which have been stated earlier (for example that Rose Street crosses the street they are driving down), and a topos that has to be inferred (if you are looking for a crossing street, and you haven't yet passed it, and there is only one crossing street left, this has to be the street you are looking for).

4 DICO is a project that aims to demonstrate how state-of-the-art spoken language technology can enable access to communication, entertainment and information services as well as to environment control in vehicles. A priority in the project is cognitive load management for safe in-vehicle dialogue. 
(5.33) a. A: Rosengatan ja det måste vara nästa Rose Streetyes it must be next

b. A: för vi kommer inte så mycket längre cause we don't get much further.

We suggest that the reason they do this is because the enthymematic structure helps the recipient of the IRU to make up her mind or correctly interpret an utterance - if the IRU provided links the assertion or suggestion made to a topos she finds acceptable she is more likely to agree with the proposition. This indicates that many artificial agents, e.g. in domains of instruction and advice giving, would communicate more efficiently if they were extended to include enthymematic competence.

These findings demonstrate the need to motivate for rhetorical purposes rather than to provide new information about one's reasons. In the context of speech interfaces for e.g. GPS systems in cars the need to justify instructions becomes even more urgent, among other reasons because human users of artificial intelligence tend to need the reassurance of explanation. This is especially true in complex tasks such as giving driving instructions based on a combination of geographical knowledge, current traffic and-possibly - the preferences of the driver. 